\title{
Feature-based search asymmetries in pigeons and humans
}

\author{
SUSAN E. ALLAN and DONALD S. BLOUGH \\ Brown University, Providence, Rhode Island
}

\begin{abstract}
Pigeon and human subjects searched for one target item amidst a number of identical distractors. Simple line forms were used. The target differed from the distractors only in terms of the presence or absence of a feature (a line or a gap); in some experimental series, the feature was present in the target; in others, the feature was in the distractors. The pigeons pecked at the target; the human subjects either reported the presence of the target or pointed to it with a light pen. The time between display onset and this response was recorded. Varied across experimental conditions were the number of distractors in the display, the nature of the stimulus forms, and certain procedural parameters; five conditions were run with pigeons and three with humans. Under all test conditions, the results from the human subjects replicated the previously reported search-asymmetry effect. That is, search speed was greater and decreased less with display size when the target bore the feature (line or gap) than when the distractors bore the feature; both yes/no and localization-response conditions yielded this effect. However, pigeons failed to show search asymmetry; neither line nor gap in a target facilitated search. The results suggest that early visual processing differs for pigeons and humans, that pigeon features differ from human features, or that search asymmetry was eliminated by the long practice given the pigeons.
\end{abstract}

There is considerable evidence that features such as line orientation, color, size, contrast, line ends, and curvature are handled somewhat independently during early visual processing in humans (e.g., see Treisman \& Gelade, 1980). As yet, however, the psychophysical analysis of such processing has been restricted almost entirely to humans. It is of interest to see whether other highly visual species show similar sorts of processing, since other species may have solved the problems of object perception in somewhat different ways. In the present experiments, we sought evidence that pigeons extract certain basic features when they perceive simple forms.

The pigeon has a highly developed visual system, and the basic visual psychophysics of this species has been extensively studied (Donovan, 1978). Pigeons discriminate simple forms readily, and their perception of form seems similar to that of humans; for example, they yield comparable similarity functions for letters of the alphabet and random dot patterns (D. S. Blough, 1982, 1984, 1985). Visual search in pigeons also appears to follow rules similar to those found in humans with respect to similarity functions, display-size functions (D. S. Blough, 1979, 1984, 1985; P. M. Blough, 1984), and texture segregation (D. S. Blough \& Franklin, 1985).

The visual search task was used here. Typically, in this task, the subject searches for a particular form, the target, on a field of other forms, the distractors. A number of experiments (Treisman \& Gormican, 1988) have shown that a salient, simple feature in a target makes the target

This research was supported in part by National Science Foundation Grant BNS-8025515. Address correspondence to: Donald S. Blough, Department of Psychology, Brown University, Providence, RI 02912. "pop out" of the display; that is, the target is quickly detected, and search time is relatively unaffected by the number of distractor items in the display. However, if the same feature is contained in the distractors, and if the target is distinguished by the absence of this feature, target detection will be slower and search reaction time (RT) will increase with the number of distractors. This difference in the pattern of search RTs with a pair of forms that differ by a single feature, depending on which form is the target and which the distractor, is called search asymmetry (Beck, 1973; Julesz, 1981; Treisman \& Souther, 1985; Treisman \& Gormican, 1988).

Search asymmetry has been used extensively by Treisman and her associates in the context of her feature-integration theory (Treisman \& Gelade, 1980). This theory suggests that visual processing occurs in two stages: preattentive and attentive. In the preattentive stage, the functions of visual processing are accomplished automatically and simultaneously for the entire visual field. In the attentive stage, a "mental spotlight" is moved from location to location; attention is focused on parts of the visual field in sequence. This general account of visual processing is rather widely favored, and related models have been proposed (e.g., see Hoffman, 1979).

It is hypothesized that the detection of targets containing a distinctive feature occurs in the preattentive stage. The second, slower, attentive stage is required for the detection of targets lacking features. But what configuration defines a "feature'? Treisman suggests that search asymmetry can be used as a diagnostic test to answer this question, and she and her associates have applied this test to a number of form pairs, using human subjects (e.g., Treisman \& Gormican, 1988; Treisman \& Souther, 1985). In a typi- 
cal test, a circle with a line through the bottom was paired with an open circle, with the line a possible feature. Subjects found the circle + line faster among circles than the other way around. Also, search RT changed little with display size for the circle + line target, but for the circle target surrounded by circles + line, RT increased linearly as a function of display size. This pattern of results defines search asymmetry, and it identifies the line as a feature.

In the present studies, humans and pigeons performed search tasks under conditions that in past research (e.g. , Treisman \& Gormican, 1988) have yielded clear evidence of search asymmetry in humans. Reaction times were recorded as a function of the presence or absence of putative features in targets or distractors, with the number of distractors in the displays a critical parameter. With pigeon subjects, we sought a pattern of search asymmetry with form types that had yielded such a pattern with human subjects in previous studies, using several task variations. We then replicated in humans the asymmetry results previously reported, using both a task requiring target localization (as required of the pigeons) and a yes/no target detection task like that employed in previous studies (Treisman \& Gormican, 1988). Finally, we explored search asymmetry in both species in an odd-item variation of the search task, in which the target on one trial might be the distractor on another.

\section{EXPERIMENT 1 PIGEON SEARCH FOR CONSISTENT TARGETS}

\section{Method}

Subjects

Three White Carneaux pigeons were maintained at approximately $80 \%$ of their free-feeding weights. All 3 birds had previous experience with the visual search task, but they had never been exposed to the stimuli used in the following experiments. Daily sessions of 2-3 h were run, during which the birds received mixed grain as a reward; this constituted their daily ration of food.

\section{Apparatus}

The pigeons worked in experimental chambers measuring $35 \times 30 \times 35 \mathrm{~cm}$. Set in one wall were a Lehigh feeder and a blackand-white TV monitor. Visual displays appeared on the face of the $5 \times 9 \mathrm{~cm}$ TV monitor and were viewed from a distance of approximately $7 \mathrm{~cm}$. The monitor was equipped with two rows of infrared emitter detectors, which recorded the horizontal component of beak location during responding. These arrangements are described in detail in D. S. Blough (1986). The experimental sessions were run by Atari 800 personal computers, which operated the feeders, produced stimulus displays, and recorded responses.

\section{Stimuli}

The stimuli were black forms displayed on a white background. The square forms were $4 \mathrm{~mm}$ on each side, and the other forms are drawn to scale in Figure 1. Four pairs of stimuli were used; the forms in each pair differed only in that one possessed a "feature" (i.e., a line or a gap), while the other lacked the same feature. The pairs comprised (1) a regular circle and a circle with a vertical line through the bottom; (2) a regular isosceles triangle and an isosceles triangle with a gap on the right side; (3) a boxed S and $a$ boxed $S$ with a line through top and bottom (dollar sign);

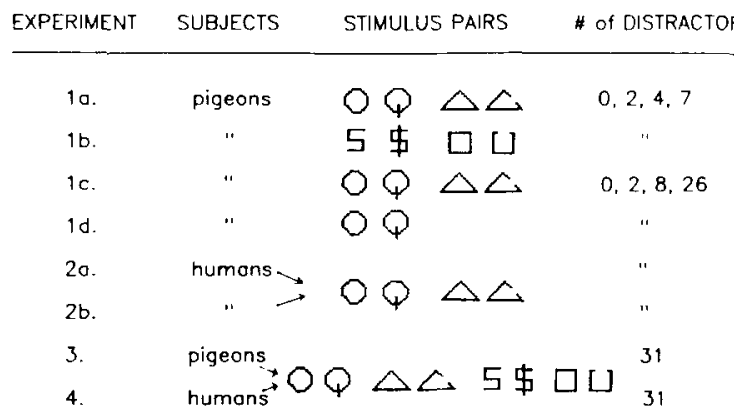

Figure 1. A summary of the experimental procedures. The square stimuli were $4 \times 4 \mathrm{~mm}$ on the display screen; the other stimuli are drawn to scale.

(4) a square and a square with a gap in the top. Only one or two of these pairs were used in any given phase of the experiments.

\section{Procedure}

Pretraining. The pigeons were first presented with displays consisting only of a single target item, without any distractors. An item from each pair was chosen as a target, such that one target possessed a feature (line or gap), while the other one did not. The pigeons were run on the two targets for several sessions, until their percents correct were in the high $90 \% \mathrm{~s}$. The pigeons were then presented with visual displays consisting of one target surrounded by identical distractors. The number of forms displayed simultaneously (display size) varied from 1 to 27 , depending on the experimental condition (see below). The pigeons' task was to peck at the target. In each condition, the pigeon was tested on two form pairs. Only forms from the same pair appeared in the same display, one as target, the other as distractor; for example, circles served as distractors for the circle + line target, and the circles + line were distractors for the circles. For each condition, each bird learned to find two targets, one containing a feature (e.g., circle + line), the other without a feature (e.g., triangle). The two possible display types (the different pairs), four display-size conditions, and target locations were all presented in random sequence, except that the number of each of these was equated across the session. The first $\mathbf{1 0}$ trials of each session served as a warm-up, with the data not recorded.

Correct responses were recorded after three consecutive pecks at the target item; the forms turned white and a blank screen flashed to give immediate feedback for a correct response. A new display followed correct responses after a 2-sec intertrial interval (ITI). During reinforcement, given for approximately $8 \%$ of the correct responses, the feeder light came on and food was presented. Incorrect responses caused the screen to turn black, and the trial was repeated until a correct response was made. The target, distractors, display size, and position of the target stayed the same on the repeated trial. The mean percent correct and RTs were calculated for every condition (the various display sizes and target types) at the end of each session. RTs for incorrect responses were not included in the calculations.

After a pigeon reached a criterion of 4 consecutive days without a trend (defined as a sequential increase or decrease in percent correct or RT), the targets and the distractors switched roles, and the procedure was run again, including one training session with the new target items.

Condition 1A. The circle and triangle forms were used in the first condition; these stimuli mimicked forms that previously had yielded strong search asymmetries in humans (Treisman \& Souther, 1985). Two birds were first trained on circle + line and triangle as targets; the 3 rd bird was first trained on circle and triangle + gap 


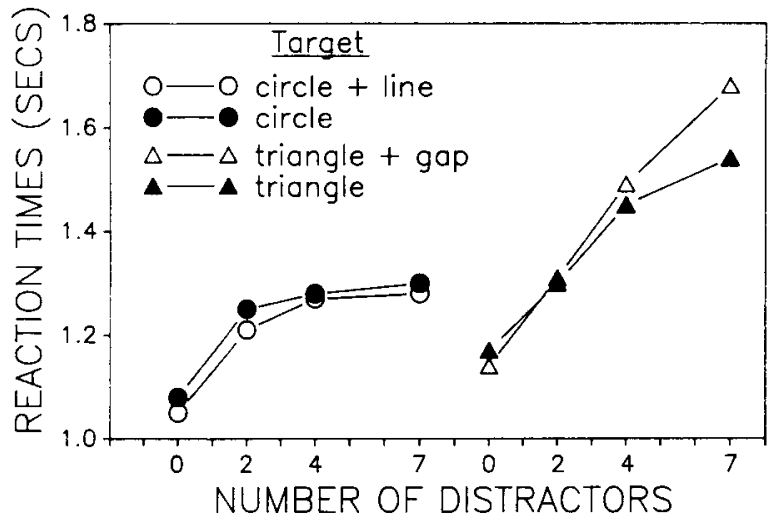

Figure 2. Experiment 1, Condition 1A: Mean reaction times to the target as a function of display size for circle and triangle pairs, smaller display sizes.

as targets. The pigeons completed 1,000 trials during a daily 2-3 h session. After the training procedure described above, the pigeons were tested on display sizes of $1,3,5$, and 8 (i.e., 1 target and $0,2,4$, or 7 distractors). The forms appeared in an irregular row on the display screen; they were displaced randomly up or down by the height of the one form. The target location was randomly assigned on each trial.

Condition 1B. This condition was the same in all respects as Condition 1A, except that the line and gap features were embedded in different forms. The $S$ pair and the square pair of stimuli were used (Figure 1).

Condition 1C. Because feature-based search asymmetry in humans is enhanced by large displays (e.g., see Treisman \& Souther, 1985), the range of the display sizes was increased. The displays contained one target and $0,2,8$, or 26 distractors. The forms were placed randomly in 32 possible locations on the screen (4 rows and 8 columns). The stimuli from Condition $1 \mathrm{~A}$ (circle and triangle pairs) were used. The ITI interval was lengthened to $3 \mathrm{sec}$ to allow time for the computer to generate the larger displays. Sessions included 800 trials.

Condition 1D. In the previouts conditions, either of two targets might appear on any trial, and this lack of predictability might have affected search strategy. Therefore, this condition contained only the circle pair of stimuli. The number of trials for each display size was doubled; otherwise the procedure duplicated that of Condition 1A. For 2 birds, the first target was a circle + line; for the 3rd, the first target was the circle.

\section{Results and Discussion}

\section{Condition 1A}

The reaction times for each bird were averaged over the 4 days before criterion, and the individual data pooled. The mean RTs appear in Figure 2. The data from each half of each stimulus pair were combined for analysis. An analysis of variance (ANOVA), with repeated measures on both factors, showed the effects of display size to be significant for the circle and the triangle pairs (see Table 1). Neither the target effect nor the interaction between target type (the presence or absence of a feature in a given form pair) and display size was significant for either form pair. One bird differed markedly from the other 2 birds in both overall speed and accuracy.

As is evident in Figure 2, RT increased with the number of distractors. This display-size effect was greatest for the triangle pair, which also yielded the lowest percent correct on the larger displays. Assuming that similarity can be defined by increases in RT and in the number of errors made, these results agree with previous reports that the display-size effect increases with target-distractor similarity (e.g., D. S. Blough, 1979; P. M. Blough, 1984). However, the presence or absence of a feature in the target did not affect the speed of the search for either the line feature or the gap.

\section{Condition 1B}

Again, RTs for the last 4 days for each bird were averaged and a combined mean was calculated. The results appear in Figure 3. An ANOVA with repeated measures on both factors indicated that search time increased as a

Table 1

ANOVA Results for Experiment 1

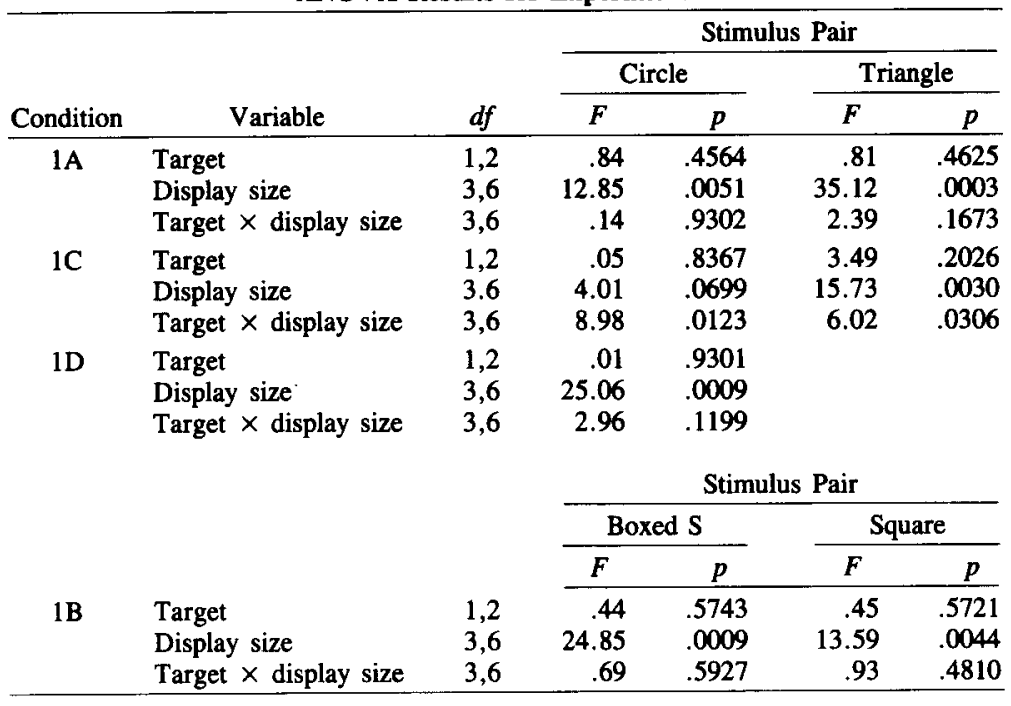




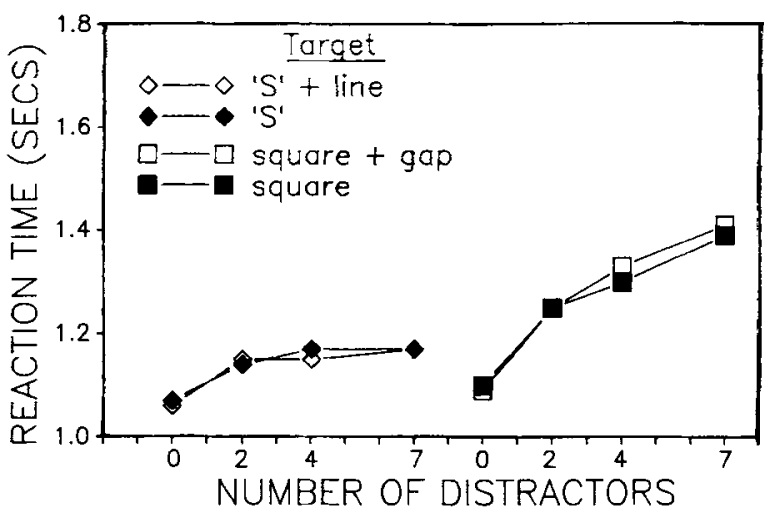

Figure 3. Experiment 1, Condition 1B: Mean reaction times to the target as a function of display size for boxed $S$ and square pairs, smaller display sizes.

function of the number of distractors for both feature pairs (see Table 1). No significant target effects or target $\times$ display-size interactions were found for either pair, and thus there was no evidence for search asymmetry. All birds showed the same general pattern of responding and had comparable percent correct scores $(93 \%-100 \%)$ for all display sizes.

\section{Condition 1C}

All birds made more errors than they had made with the smaller display sizes in the first two conditions. Mean RTs as a function of display size are shown in Figure 4 for both target pairs. An ANOVA with repeated measures indicated no target effect or effect of display size for the circle pair. A significant interaction between display size and the target type was found (see Table 1). However, the pattern of responses varied widely across birds.

The ANOVA on the triangle pair showed an effect of display size and a significant interaction of display size and target effect. No target effect was found (see Table 1). The interaction suggested a feature effect, but, as Figure 4 indicates, this was opposite in direction to that found in human data; reaction times for the target possessing the hypothesized feature (the triangle + gap) increased more rapidly with increasing display sizes than did the target lacking the feature (triangle). All birds showed this difference, which was also in the same direction as the nonsignificant difference with triangle pairs in Condition 1A (Figure 2, rightmost points). These results suggest that the stimulus for a "feature" differs for pigeons and humans, at least in the case of the triangle, with pigeons responding to "connectedness" or "closure" as a feature.

\section{Condition 1D}

Mean RTs for each bird were determined for the last 2 days of testing, and then combined. The overall mean RTs as a function of display size for each target are shown in Figure 5. The data from each target condition were combined for analysis. An ANOVA, with repeated measures, was run on the data. The effect of display size was significant (see Table 1). Neither the target effect nor the interaction between the target type and the display size was significant. Though the levels of significance shifted somewhat from Condition 1C to ID (Table 1), the patterns of results from the two tests (Figures 4 and 5) differ too little to suggest a meaningful difference in outcome. This conclusion is consistent with the results of $\mathbf{P}$. $\mathbf{M}$. Blough (1984), who found that search for two targets was as fast as search for one.

\section{EXPERIMENT 2 \\ HUMAN SEARCH FOR CONSISTENT TARGETS}

The purpose of Experiment 1 was to seek visual search asymmetries that might define critical features for pigeons. The features tested seemed comparable to those that have elicited large effects in humans (e.g., Treisman \& Souther, 1985), but they produced no asymmetries in the pigeons, except for a small RT shift in the nonpredicted direction. Perhaps procedural differences account for this failure to replicate human results. A major difference between the task used here and that used by Treisman and

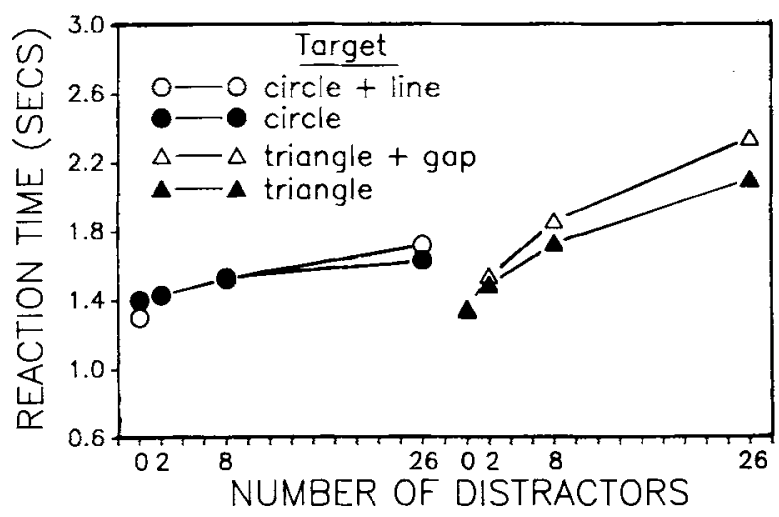

Figure 4. Experiment 1, Condition 1C: Mean reaction times to the target as a function of display size for circle and triangle pairs, larger display sizes.

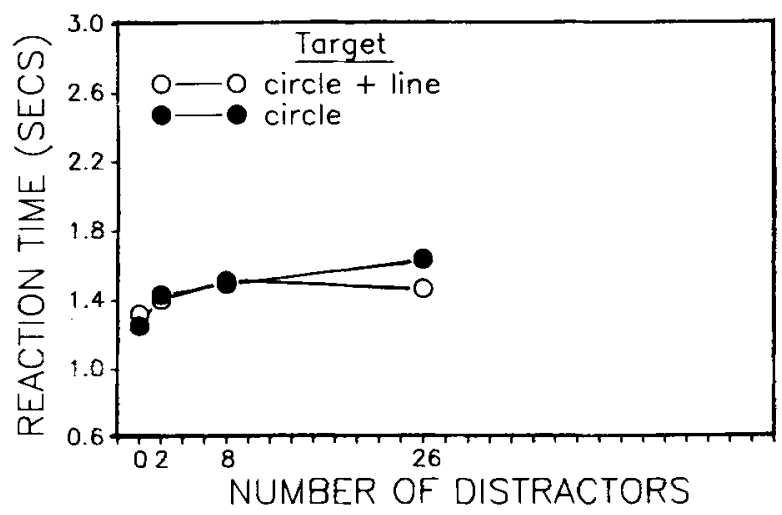

Figure 5. Experiment 1, Condition 1D: Mean reaction times to the target as a function of display size for circle pairs presented unmixed with other pairs. 
her associates was that the pigeons responded by pecking at the target, thus locating it, whereas human subjects typically have indicated only whether or not a target was present in the display. Treisman has suggested that localizing a target requires focused attention. If this were true, the short RT that should result from the "pop-out" of a simple feature could be lost by the conjunction of feature detection with the localization process. Thus, the localization requirement in Experiment 1 could have obscured search asymmetries. To check this possibility, human subjects in Experiment 2 were required to localize targets in tasks similar to those we used with the pigeons.

\section{Method}

\section{Subjects}

Five graduate students and 1 medical student volunteered as subjects. Every subject completed the experiment in one 1-h session and served in all conditions.

\section{Apparatus}

The apparatus and procedure were as in Experiment 1, except for modifications adapting them for human subjects. Stimuli appeared on a monochromatic $13 \times 18 \mathrm{~cm}$ display monitor. Subjects responded by touching the display with a Tech-Sketch Light Pen, which plugged into the Atari keyboard.

\section{Stimuli}

The same forms were used as in Condition 1A. They were $6 \times 6 \mathrm{~mm}$ (based on the square form) and were viewed from a distance of approximately $40 \mathrm{~cm}$.

\section{Procedure}

The subjects were run for two 240-trial blocks, with a 5-min break between blocks. The search task was identical to that of Experiment 1 , Condition $1 \mathrm{C}$, and it employed the same target and distractor pairs (Figure 1); the numbers of distractors varied among $0,2,8$, and 26 .

Condition $2 \mathrm{~A}$. The subjects sat before the screen, at a comfortable reading distance of approximately $40 \mathrm{~cm}$, and they were instructed to touch the target on the screen with the light pen. They were told to respond as quickly as possible without sacrificing accuracy. Before the start of the first block, the subjects were shown a drawing of the two targets they should search for. On each trial, as in Experiment 1, Condition $1 \mathrm{C}$, one target was present together with its paired distractor. One circle pair and one triangle pair were intermixed during the first test block; the alternative circle and triangle pairs were intermixed during the second block. Which targets were used first and which second was counterbalanced across subjects. Ten warm-up trials were run before each block to familiarize the subjects with the targets and the light pen.

A correct response was signaled by the forms flashing white, followed by a 2-sec ITI. An incorrect response was followed by a brief blackout period, after which the trial was repeated until a correct response was made. The target, distractors, display size, and position of the target stayed the same on the repeated trial. Incorrect responses were not included in the $\mathbf{R T}$ data.

Condition 2B. In order to ensure that the results were not in some way unique to the conditions under which the experiment was run, the experiment was rerun in a yes/no format typical of previous search-asymmetry demonstrations. The displays were as in the previous condition, except that on half the trials the target was not present. The subjects were again shown a sketch of the two targets they were to search for. Instructions were as before, except that the subjects used a "joy stick" to indicate whether or not a target was present, pushing up for "yes" and down for "no."

\section{Results and Discussion}

\section{Condition 2A}

Figure 6 shows the mean RTs for all subjects across both blocks. ANOVAs generally supported the results evident in the figure. A target effect was not significant for the circle pair, though both display size and the interaction (target $\times$ display size) were significant. For the triangle pair, the analysis showed a significant target effect, display-size effect, and interaction (see Table 2). The subjects showed the same pattern of responding, but their overall speeds varied.

The display-size $\times$ target-type interaction was highly significant for each group of targets. Thus, asymmetries in search were evident for targets that differed in the presence or absence of a feature, indicating that the localization requirement does not eliminate search asymmetry. Because the localization condition made the human task more like that given the pigeons, the results also support the idea that pigeons differ from humans in the visual processing of these forms. Pigeons may perceive different salient features than humans do, or features may not play a comparable role in early detection under these conditions. We will discuss these issues further below.

\section{Condition 2B}

The mean RTs for the circle pair are shown in Figure 7. An ANOVA showed a significant target effect and an effect of display size. The difference between trial types, yes and no trials (whether a target was present or not), was significant. The two-way interaction between the type of target and the display size was significant, as was the interaction between target type and trial type. The twoway interaction of display size and trial type was also significant. The three-way interaction of target type, trial type, and display size was significant (see Table 2).

Similar results were obtained for the triangle pair. The mean RTs as a function of display size are shown in Figure 8. All three main effects, target type, display size, and trial type, were significant. The two-way interactions of target type and display size, target type and display

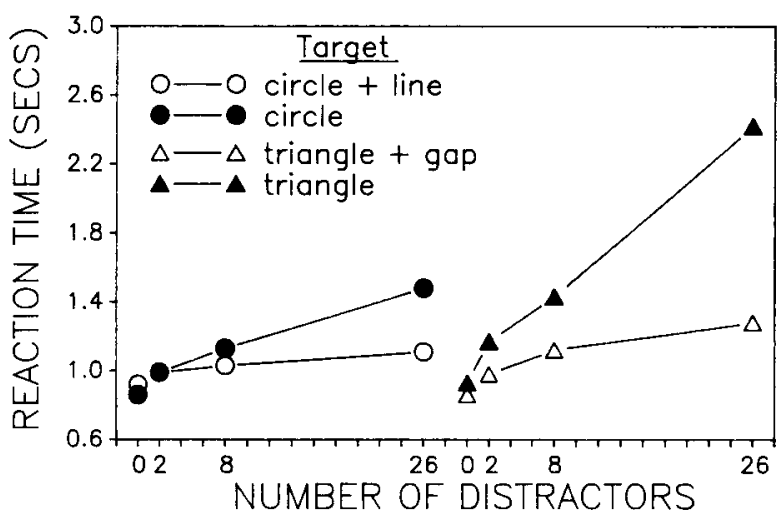

Figure 6. Experiment 2, Condition 2A: Mean reaction times to the target as a function of display size for human subjects run on the location task with circle and triangle pairs. 
Table 2

ANOVA Results for Experiment 2

\begin{tabular}{|c|c|c|c|c|c|c|}
\hline \multirow[b]{3}{*}{ Condition } & \multirow[b]{3}{*}{ Variable } & \multirow[b]{3}{*}{$d f$} & \multicolumn{4}{|c|}{ Stimulus Pair } \\
\hline & & & \multicolumn{2}{|c|}{ Circle } & \multicolumn{2}{|c|}{ Triangle } \\
\hline & & & $\boldsymbol{F}$ & $p$ & $F$ & $p$ \\
\hline $2 A$ & $\begin{array}{l}\text { Target } \\
\text { Display size } \\
\text { Target } \times \text { display size }\end{array}$ & $\begin{array}{l}1,5 \\
3,15 \\
3,15\end{array}$ & $\begin{array}{r}2.55 \\
35.01 \\
11.59\end{array}$ & $\begin{array}{l}.1711 \\
.0001 \\
.0003\end{array}$ & $\begin{array}{l}42.64 \\
59.94 \\
52.67\end{array}$ & $\begin{array}{l}.0013 \\
.0001 \\
.0001\end{array}$ \\
\hline 2B & $\begin{array}{l}\text { Target } \\
\text { Display size } \\
\text { Yes } / \text { no trials } \\
\text { Target } \times \text { display size } \\
\text { Target } \times \text { yes } / \text { no } \\
\text { Display } \times \text { yes } / \text { no } \\
\text { Target } \times \text { display size } \\
\times \text { yes } / \text { no }\end{array}$ & $\begin{array}{l}1,5 \\
3,15 \\
1,5 \\
3,15 \\
1,5 \\
3,15\end{array}$ & $\begin{array}{l}20.75 \\
20.09 \\
25.07 \\
23.93 \\
19.89 \\
13.39\end{array}$ & $\begin{array}{l}.0061 \\
.0001 \\
.0041 \\
.0001 \\
.0066 \\
.0002\end{array}$ & $\begin{array}{l}44.42 \\
54.79 \\
51.53 \\
56.46 \\
13.95 \\
23.92\end{array}$ & $\begin{array}{l}.0011 \\
.0001 \\
.0008 \\
.0001 \\
.0135 \\
.0001\end{array}$ \\
\hline
\end{tabular}

type, and display size and trial type were also all significant. The three-way interaction again was significant (see Table 2). As in Condition 2A, the data from individual subjects were consistent with the pattern of responding seen in the mean data.

The results show strong asymmetries in search, depending on the presence or absence of a feature in a target item. Responses to negative displays were slower for all targets, though much greater for the targets lacking a feature. These results all replicate the results of previous experiments for similar displays (Treisman \& Gormican, 1988; Treisman \& Souther, 1985). If a target possesses a feature, its presence is signaled by "pop-out" from the display, and its absence is more rapidly determined than when it has no salient feature.

\section{EXPERIMENT 3 PIGEON SEARCH FOR THE ODD ITEM}

Experiment 3 involved different pigeons in a new search task, in another attempt to induce search asymmetry. The task was a slightly modified version of the odd-item task described by D. S. Blough (1986). As above, displays contained one target item surrounded by identical distractor items. However, in these odd-item experiments, the target on one trial could be the distractor on the next. Thus, the targets were not defined as specific items, but rather as the unique items in the displays. All four stimulus pairs used in Experiments 1 and 2 appeared during each session, and each member of a pair appeared sometimes as target and sometimes as distractor. However, as before, an individual display contained only the two members of a single pair that differed in terms of the presence or absence of a feature.

\section{Method}

Subjects

The subjects were 3 White Carneaux pigeons that had not been used in the previous experiments. They were maintained at $80 \%$ free-feeding weight. The birds had a short history of training on an odd-item search task, though not with the same stimuli as those used here.

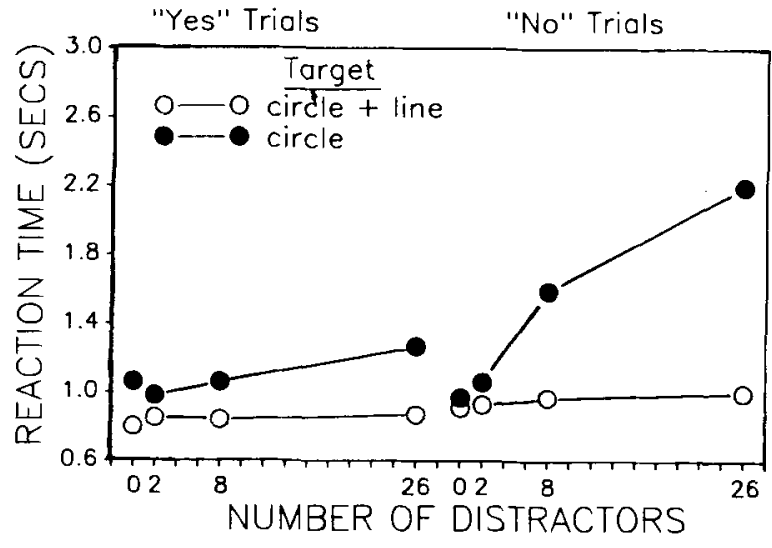

Figure 7. Experiment 2, Condition 2B: Data from circle pairs only. Mean reaction times to the target as a function of display size for human subjects run on the yes/no task. On the left are reaction times for positive (yes) trials; on the right are reaction times for negative (no) trials.

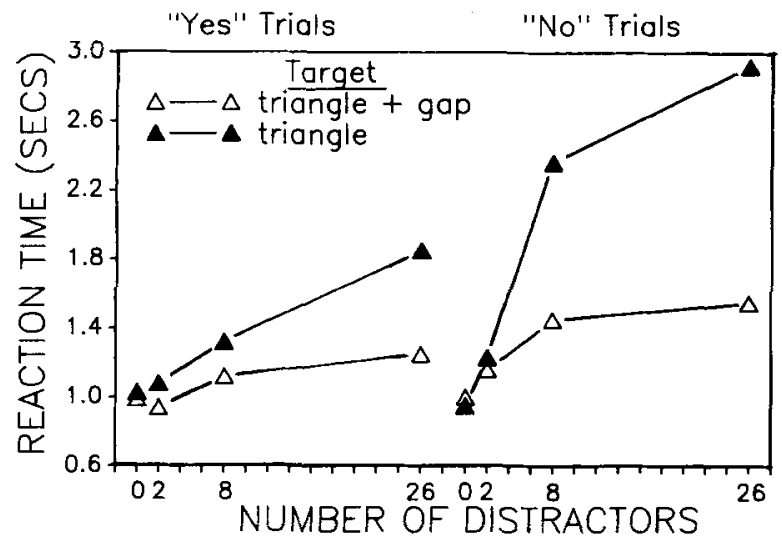

Figure 8. Experiment 2, Condition 2B: Data from triangle pairs only. Mean reaction times to the target as a function of display size for human subjects run on the yes/no task. On the left are reaction times for positive (yes) trials; on the right are reaction times for negative (no) trials. 


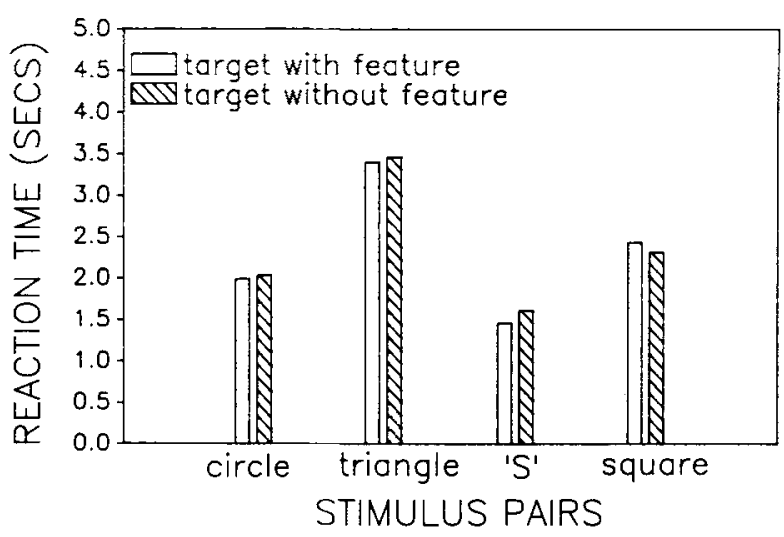

Figure 9. Experiment 3. Mean reaction times for four stimulus pairs on the odd-item task with pigeons. The bars distinguish the target + feature displays from the target without feature displays.

\section{Apparatus and Stimuli \\ The apparatus and the four stimulus pairs from the previous ex-} periments were used (see Figure 1).

\section{Procedure}

Pretraining. The birds were trained to peck at a black form on the screen. After responding readily, they were presented with the forms to be used in the experimental session, each form appearing alone on the screen. Four sessions of 800 trials each were run, exposing the birds to each form for 400 trials.

Experimental sessions. The birds were presented with displays of 32 items arranged in a horizontal $4 \times 8$ matrix. The target was randomly assigned to any 1 of the 12 inside positions in the matrix, and the paired distractor appeared in all the other positions. The pigeons ran either 960 or 1,120 trials during a daily session, which lasted 2-3 h. Each session began with 8 warm-up trials, during which each stimulus appeared once without distractors. Randomly arranged in each subsequent block of 16 trials were 2 trials for each of the eight possible display types (four stimulus pairs, with each member of a pair serving once as target and once as distractor). The sequence of events during a trial and the reinforcement probabilities were as described in Experiment 1. Mean RTs for each display type and an overall percent correct were calculated after every session. The birds were run for 5 days after a criterion of 4 consecutive days without a trend, making a total of 9 days for analysis.

\section{Results and Discussion}

The mean RTs for the four stimulus pairs are shown in Figure 9. An ANOVA was run on each stimulus pair. No significant target effects were found (see Table 3). The pigeons responded with approximately the same speed, whether the target possessed a distinguishing feature or not. Accuracy was between $80-90 \%$ for all subjects, with little individual variation in the pattern of responding.
These results support the previous finding that features as defined here did not play a role in the pigeons' visual processing of the displays.

\section{EXPERIMENT 4 HUMAN SEARCH FOR THE ODD ITEM}

\section{Method}

\section{Subjects}

Six graduate students served as subjects. Three of the 6 served as subjects for Experiment 2.

\section{Apparatus and Stimuli}

The apparatus was that used in Experiment 2. The stimuli were the 4 pairs that were used with pigeons in Experiment 3.

\section{Procedure}

The procedure followed that of Experiment 3, with the adaptations for human subjects described in Experiment 2. Four hundred trials, consisting of 25 blocks of 16 trials, were run in a single .5-h session. Two presentations of the eight display types were randomized within each block.

\section{Results and Discussion}

An ANOVA was run on mean RTs for each stimulus condition for each subject. A significant target effect was found for the circle pair, the triangle pair, and the square pair (see Table 3). There was no significant difference in RTs to the targets in the boxed S pair (see Figure 10).

The target effects were all in the predicted directions. When the target possessed a feature and the distractors did not, search speed was significantly faster than when the target lacked the feature. This result shows that search asymmetry does not depend on having a consistent target or an expectation as to which form the target will be. Only the boxed $\mathrm{S}$ pair failed to show this feature effect. Since each member of the pair mimicked a very familiar form ( $\mathrm{S}$ and dollar sign), it is possible that their distinctiveness to humans eclipsed any specific feature effect. A familiarity effect is suggested also by the fact that search was faster for this pair than for any other.

\section{GENERAL DISCUSSION}

Our results suggest that the processing of features in visual search may differ in pigeons and humans. Two experiments replicated the search-asymmetry phenomenon in humans, and extended these findings by demonstrating search asymmetry under the localization and odd-item conditions. In contrast, two experiments involving two

Table 3

ANOVA Results for Experiments 3 and 4

\begin{tabular}{|c|c|c|c|c|c|c|c|c|c|c|}
\hline \multirow[b]{3}{*}{ Experiment } & \multirow[b]{3}{*}{ Variable } & \multirow[b]{3}{*}{$d f$} & \multicolumn{8}{|c|}{ Stimulus Pair } \\
\hline & & & \multicolumn{2}{|c|}{ Circle } & \multicolumn{2}{|c|}{ Triangle } & \multicolumn{2}{|c|}{$\mathbf{S}$} & \multicolumn{2}{|c|}{ Square } \\
\hline & & & $F$ & $p$ & $\boldsymbol{F}$ & $p$ & $F$ & $p$ & $F$ & $p$ \\
\hline $\begin{array}{l}3 \text { birds } \\
4 \text { humans }\end{array}$ & $\begin{array}{l}\text { Target } \\
\text { Target }\end{array}$ & $\begin{array}{l}1,2 \\
1,5\end{array}$ & $\begin{array}{r}.34 \\
10.04\end{array}$ & $\begin{array}{l}.6199 \\
.0249\end{array}$ & $\begin{array}{r}.81 \\
42.65\end{array}$ & $\begin{array}{l}.4629 \\
.0013\end{array}$ & $\begin{array}{r}2.28 \\
.57\end{array}$ & $\begin{array}{l}.2699 \\
.4840\end{array}$ & $\begin{array}{r}1.09 \\
112.14\end{array}$ & $\begin{array}{l}.4056 \\
.0001\end{array}$ \\
\hline
\end{tabular}




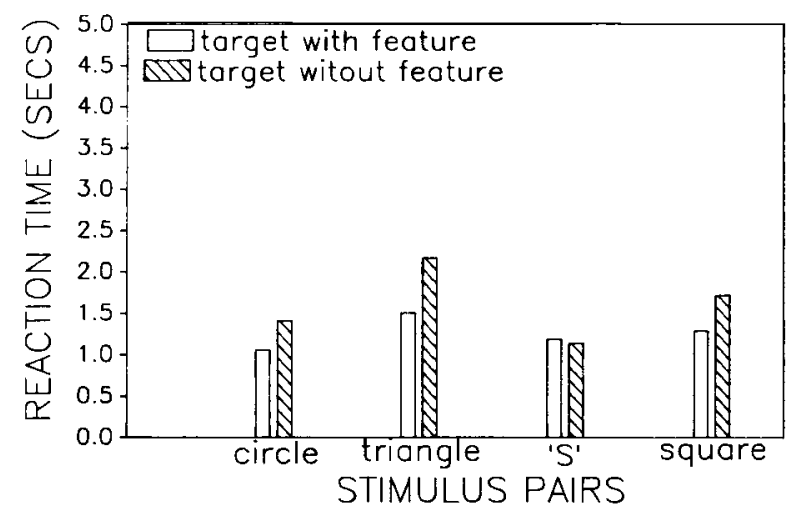

Figure 10. Experiment 4. Mean reaction times for four stimulus pairs on the odd-item task with humans. The bars distinguish the target + feature displays from the target without feature displays.

groups of pigeons in five experimental conditions revealed almost no evidence of search asymmetry. One small and variable effect in the expected direction appeared in one condition, but another condition produced a more robust effect in the opposite direction (i.e., the absence of a gap in the target, rather than its presence, facilitated search).

How may the pigeon-human difference be understood? It could reflect a basic difference in the processes underlying form recognition in the two species. However, previous research has suggested that these processes may function comparably in important respects. As noted earlier, the pattern of similarity between simple forms is remarkably alike for the two species (e.g., see D. S. Blough, $1982,1984,1985$ ), and visual search exhibits similar functional relations. In particular, for both species, RT/displaysize functions are relatively flat for distinctive targets, and increase in steepness with target-distractor similarity (D. S. Blough, 1979; P. M. Blough, 1984). Of course, despite this evidence of comparable processing in other tasks, search asymmetry may reflect basic differences. It might mean, for example, that pigeons process these simple forms in a holistic way that does not depend on early feature extraction.

A fundamental difference in visual processing is, however, not the only possible explanation of the pigeonhuman difference described above. Among the more interesting alternatives is the idea that search may be similarly influenced by features in the two species, but that pigeons use different features than humans do. We note that we tested features in only a few forms. Although our data agree with others', in that a gap is a feature for humans, some of our data (Experiment 1 triangle results) hint that connectedness (the absence of a gap) may be a feature for pigeons. Also, feature-based asymmetries often may not be as striking as our human results. The recent compilation of feature asymmetries by Treisman and Gormican (1988) shows considerable variability in the size of effects; for example, target-distractor combinations incorporating features that differed on a quantitative dimension (e.g., line tilts of different degrees) showed search asymmetries less pronounced than those found for salient qualitative features such as line end and gap. Thus, it is possible that further exploration will turn up feature effects in pigeons, and it would be of special interest to test quantitative dimensions in this species.

A third source of the pigeon-human difference could be procedural, and it is of course impossible to eliminate all procedural differences in cross-species comparisons. A difference we did explore was the target-localization requirement, which was imposed on the pigeons but is not usually imposed on human subjects. However, our human subjects still showed robust search asymmetry when required to localize targets. This finding is of interest in its own right. The feature-integration theory of Treisman and Gelade (1980) indicates that localization requires focused attention. This presumably is true whether or not the search target is characterized by feature presence or by feature absence, so localization should tend to equalize the attentional requirements of these conditions. Since the theory suggests that search asymmetry reflects differential attentional requirements, one might expect the effect to be eliminated by the localization requirement. Since this was not the case, the data suggest that localization occurs at a stage of search different from detection (cf. Treisman \& Gormican, 1988).

Another procedural difference between the pigeon experiments and most human experiments, including ours, could be crucial: Pigeons and humans received grossly different amounts of training prior to the test sessions. Our human subjects saw drawings of the stimulus pairs, responded on eight warm-up trials, and then began their experimental runs. In contrast, the sessions used to train the pigeons involved thousands of trials prior to final testing. This extensive training might well have altered search processing. There is much evidence that, in humans, search becomes automatized with extensive practice (e.g., see Schneider \& Shiffrin, 1977). It could be argued that the humans had prior exposure to the forms used in the experiment, but this seems clearly the case only with the $\$$ form. Though the pigeons did not have such preexperimental exposure, they received much more practice in the experiment than the humans did. It would be interesting to explore the effect of practice on search asymmetry in humans. The theories of Treisman (e.g., Treisman \& Gelade, 1980) and related views of others (e.g., Julesz, 1981) imply that such effects are the result of early processing that may be "prewired" in the visual system. If this is so, they would seem relatively immune to effects of experience.

Less interesting procedural differences between the pigeon and human experiments could have affected the results. For example, viewing conditions differed considerably in an absolute sense: though variation was less in relative terms, the visual angle subtended by single forms was about three times as large for pigeons $\left(3.3^{\circ}\right)$ as it was for humans $\left(.86^{\circ}\right)$. This may compensate for the somewhat lower visual acuity of pigeons than of humans under these conditions (Hodos, Liebowitz, \& 
Bonbright, 1976); in any event, the forms were readily discriminated by both pigeons and humans.

Some readers will doubtless notice a parallel between search asymmetry and the feature-positive effect, a phenomenon of visual discrimination strikingly demonstrated by pigeons (e.g., see Jenkins, 1973). When two discriminative stimuli differ in terms of the presence of a feature on one stimulus (say, a red dot on a white key) and the absence of this feature on the other (say, a blank white key), the discrimination is learned rapidly if reward is associated with the feature stimulus and nonreward with the blank stimulus. In contrast, discrimination is much slower, and it may fail entirely, if reward is associated with the stimulus lacking the feature. Thus, the featurepositive effect is like the search-asymmetry effect, because in both cases performance is enhanced when the reinforced form (the target, in search) contains a distinctive feature.

Because the feature-positive effect is so marked in pigeons, it may seem strange that search asymmetry is not also observed. The difference may lie in the functional definition of the "feature." In the sense implied by previous feature-positive research, the entire target form is the "distinguishing feature" in all the search displays used here. This identification is all the more persuasive, because observers of the feature-positive effect report that pigeons invariably peck at the distinctive feature (e.g., the red dot) in the feature-positive case. Thus, it is likely that all of our search displays corresponded to the featurepresent stimulus in ordinary discrimination learning; since no feature-absent displays were used, we would not expect a differential effect from this source.

In summary, we have replicated and extended the evidence for search asymmetries in humans, but we have largely failed to find these asymmetries in pigeons. Among the possible causes for this apparent species difference are: (1) a fundamental difference in the way that the two species process simple line forms during search; (2) the use of inappropriate test forms with the pigeons, which may respond to different features than do humans; (3) the large amount of practice given the pigeons, which may have attenuated effects that might be present at the early stage; (4) other unspecified differences in viewing conditions or experimental requirements, which must always be large in research with such different species. We advocate further research on the second and third of these possibilities, since it would be of considerable interest to identify a family of "pigeon features" and/or to discover substantial effects of practice on the definition of a functional feature.

\section{REFERENCES}

BECK, J. (1973). Similarity grouping of curves. Perceptual \& Motor Skills, 36, 1331-1341.

BLough, D. S. (1979). Effects of the number and form of stimuli on visual search in pigeons. Journal of Experimental Psychology: Animal Behavior Processes, 5, 211-223.

BLough, D. S. (1982). Pigeon perception of letters of the alphabet. Science, 218, 297-298.

Blough, D. S. (1984). Form recognition in pigeons. In H. L. Roitblat, T. G. Bever, \& H. S. Terrace (Eds.), Animal Cognition (pp. 277289). Hillsdale, NJ: Erlbaum.

BLough, D. S. (1985). Discrimination of letters and random dot patterns by pigeons and humans. Journal of Experimental Psychology: Animal Behavior Processes, 11, 261-280.

BLough, D. S. (1986). Odd-item search by pigeons: Method, instrumentation, and uses. Behavior Research Methods, Instruments, \& Computers, 18, 413-419.

Blough, D. S., \& Franklin, J. J. (1985). Pigeon discrimination of letters and other forms in texture displays. Perception \& Psychophysics, 38, 523-532.

Blough, P. M. (1984). Visual search in pigeons: Effects of memory set size and display variables. Perception \& Psychophysics, 35, 344-352.

Donovan, W. J. (1978). Structure and function of the pigeon visual system. Physiological Psychology, 6, 403-437.

Hodos, W., Leibowitz, R. W., \& BonbriGht, J. C. (1976). Nearfield visual acuity of pigeons: Effects of head location and stimulus luminance. Journal of the Experimental Analysis of Behavior, 25, 129-141.

Hoffman, J. E. (1979). A two-stage model of visual search. Perception \& Psychophysics, 25, 319-327.

JeNkINs, H. M. (1973). Noticing and responding in a discrimination based on a distinguishing element. Leaming \& Motivation, 4, 115-137.

JuLesz, B. (1981). Textons, the elements of texture perception, and their interactions. Nature, 290, 91-97.

SCHNEIDER, W., \& SHFFrIN, R. M. (1977). Controlled and automatic human information processing: I. Detection, search, and attention. Psychological Review, 84, 1-66.

Treisman, A., \& GelaDE, G. (1980). A feature-integration theory of attention. Cognitive Psychology, 12, 97-136.

Treisman, A., \& Gormican, S. (1988). Feature analysis in early vision: Evidence from search asymmetries. Psychological Review, 95, $15-48$.

Treisman, A., \& SoUTHER, J. (1985). Search asymmetry: A diagnostic for preattentive processing of separable features. Journal of $E x$ perimental Psychology: General, 114, 285-310.

(Manuscript received November 7, 1988; revision accepted for publication May 1, 1989.) 